\title{
La alimentación desde una perspectiva multidimensional en la formación de docentes en ejercicio
}

\author{
A multidimensional perspective of "Feeding" \\ in a pedagogical experience of in-service teacher education
}

\author{
Silvina Cordero ${ }^{1}$ - Adriana Mengascini ${ }^{2}$. Adriana Menegaz ${ }^{3}$. \\ Mariel Zucchi ${ }^{4}$. Ana Dumrauf ${ }^{4}$
}

\begin{abstract}
Resumen: Este trabajo describe y analiza las características y resultados de una experiencia pedagógica de formación de docentes de Educación Infantil en ejercicio, cuyo eje temático fue Alimentación desde una perspectiva compleja y multidimensional. Se presenta una revisión del tratamiento de esta temática en el campo de la educación en Ciencias Naturales, se describe y fundamenta la experiencia y se enmarca metodológicamente el análisis. Finalmente se comentan resultados que evidencian la revisión de la noción escolarizada de Alimentación y su complejización en proyectos educativos situados. Contribuimos así al campo de la formación de docentes en Ciencias Naturales, Ambiente y Salud, a la par que avanzamos en la comprensión de nuestra propia práctica como docentes formadoras.
\end{abstract}

Palabras clave: Formación docente. Educación infantil. Alimentación. Investigación-acción. Ciencias naturales, ambiente y salud.

\begin{abstract}
This work describes and analyzes the characteristics and outcomes of a pedagogical experience in teacher education, whose focus was "Feeding" from a complex and multidimensional perspective. A review of the treatment of this subject in the field of education in Natural Sciences is presented. The characteristics and foundations of the pedagogical experience are exposed, and the analysis is methodologically framed. Results show the revision of the school notion of "Feeding" and the elaboration of more complex and located educational projects. In this way, we contribute to the field of teacher education in Natural Sciences, Environment and Health, and at the same time we improve our understanding of our own practice as teacher trainers.
\end{abstract}

Keywords: Teacher training. Childhood education. Feeding. Action-research. Natural sciences, environment and health.

\footnotetext{
${ }^{1}$ Universidad Nacional de La Plata (UNLP), Instituto de Investigaciones en Humanidades y Ciencias Sociales, Facultad de Humanidades y Ciencias de la Educación, La Plata, Buenos Aires, Argentina. E-mail: < silvina. cordero.protto@gmail.com>

${ }^{2}$ Universidad Nacional de Luján, Departamento de Educación, Buenos Aires, Argentina.

${ }^{3}$ Universidad Nacional Arturo Jauretche, Buenos Aires, Argentina.

${ }^{4}$ Consejo Nacional de Investigaciones Científicas y Técnicas, Buenos Aires, Argentina.
} 


\section{Introducción}

La investigación en la formación de docentes en temáticas de las Ciencias Naturales, el Ambiente y la Salud (CNAS) constituye una de las líneas de trabajo que, desde el Grupo de Didáctica de las Ciencias (GDC), Universidad Nacional de La Plata-Consejo Nacional de Investigaciones Científicas y Técnicas (CONICET), Argentina, venimos desarrollando desde los años 1990. Desde esa época nos planteábamos como desafío elaborar, poner en práctica y sistematizar propuestas de formación que valoraran los saberes docentes en la enseñanza de las CNAS, reconocieran la complejidad de su práctica y que, a partir de la ampliación de su universo conceptual y didáctico, apuntaran a la reflexión y a la construcción conjunta de propuestas superadoras. Consideramos que, en este proceso, los saberes docentes se articularían con el saber experto, con las experiencias desarrolladas por colegas y se nutrirían de la teoría y la investigación educativa. Apuntamos así al desarrollo profesional docente, que se produce al construir conocimiento situado relativo a la práctica, al trabajar en el contexto de comunidades docentes, teorizar sobre su trabajo y articular con el análisis de lo social, cultural y político más amplio. Esta propuesta supone que profesores y profesoras asumen, desde una posición de autonomía, su responsabilidad en la construcción de un proyecto educativo democratizador de las relaciones, basado en el respeto a la diversidad y en la confianza en la capacidad de aprendizaje de los y las estudiantes.

En ese marco de intenciones y supuestos, este trabajo describe y analiza las características y resultados de un curso sobre la temática alimentación desde una perspectiva multidimension$\mathrm{al}^{5}$, destinado a docentes en ejercicio en la Educación Infantil ( 0 a 5 años), e implementado en 2013. Nuestro objetivo fue indagar cómo y en qué medida las cursantes se apropiaron de la perspectiva planteada y qué elementos de la propuesta formativa resultaron útiles para su trasposición a sus aulas.

A continuación contextualizamos el interés por el tratamiento de esta temática en la Educación en Ciencias; describimos y fundamentamos nuestra propuesta didáctica; presentamos los procedimientos metodológicos utilizados y exponemos algunos resultados.

\section{La alimentación en la literatura del campo de la educación en Ciencias Naturales}

En su revisión de la literatura respecto a la alimentación, Rivarosa y De Longhi (2012) plantean que, en general, los abordajes atienden a la nutrición, el aparato digestivo y aspectos fisiológicos, haciendo a veces referencia a hábitos cotidianos, higiene o consumo.

Membiela y Cid (1998) desarrollan el diseño, puesta en práctica y evaluación de una unidad didáctica para la enseñanza secundaria obligatoria, iniciando el proceso con la indaga-

\footnotetext{
${ }^{5}$ Acreditado por la Dirección de Capacitación de la Dirección General de Cultura y Educación (Provincia de Buenos Aires, Argentina). Esta dependencia gubernamental se ocupa, entre otros aspectos, de la formación continua de los y las docentes del sistema educativo provincial.
} 
ción de ideas previas sobre alimentación, entendida como "la forma y manera de proporcionar al cuerpo humano las sustancias que le son indispensables para mantener la salud y la vida" (MEMBIELA; CID, 1998, p. 500). Según estos autores, el ámbito de la enseñanza de ciencias se ha limitado habitualmente a la nutrición, es decir, a los aspectos fisiológicos y bioquímicos sin contemplar los componentes sociales o culturales (económicos, antropológicos, sociológicos, históricos). Su propuesta plantea atender a dichos componentes, pretendiendo además formar consumidores críticos, con actitudes solidarias y responsables. Más allá de estos objetivos, de la lectura de las actividades propuestas surge fundamentalmente una intencionalidad normativa hacia dietas equilibradas, la importancia del consumo de fibra alimentaria y la solidaridad hacia el problema del hambre en el mundo.

Roset Elías y Viladot Aguayo (2004) se refieren a la Educación Nutricional, definiéndola como el conjunto de actuaciones que tienden a modificar conocimientos, actitudes y comportamientos. Presentan una propuesta didáctica dirigida principalmente al primer ciclo de educación secundaria española, y consideran que la adquisición de hábitos y comportamientos saludables sería la finalidad última de la educación nutricional.

Del Campo, Vara Messler y Navarro (2010) desarrollan propuestas sobre Educación Alimentaria-Nutricional, en tanto alternativa para contribuir a la prevención y control de enfermedades relacionadas con la alimentación en una escuela primaria. Fundamentan la importancia de trabajar con niños/as parala adquisición de patrones alimentarios saludables y perdurables, en tanto consumidores actuales y futuros. De marcado sesgo normativo, tanto el diagnóstico como el desarrollo de actividades de formación para docentes enfocan en hábitos considerados recomendables por ser saludables.

Rivarosa y de Longhi (2012), desde una mirada compleja, presentan y evalúan propuestas didácticas enmarcadas en la alfabetización científica, destinadas a estudiantes secundarios. Consideran cinco ejes de trabajo: culturas y estilos de vida de ayer y de hoy; prácticas y modelos productivos; pautas culturales y costumbres familiares; cuidado del cuerpo; y alimentación vs hambre en el mundo y en el contexto nacional-regional.

Galvão y Praia (2009) analizan un proceso de formación de profesores de $2^{\circ}$ ciclo de Enseñanza Fundamental en base a estrategias de enseñanza por investigación para el tema alimentación humana. Se plantea el tratamiento interdisciplinar de la temática dentro del ámbito de la educación ciudadana, orientada al desarrollo de la salud individual y colectiva. Como resultados del proceso, los autores muestran que las actitudes de las tres profesoras involucradas en la investigación fueron adecuadas en los momentos de reflexión y planificación conjunta de propuestas, lo que indicó avances en los procesos de aprendizaje y formación, aunque no hayan motivado la reestructuración esperada en el momento de la implementación. Contrariamente a lo esperado, las profesoras mostraron una tendencia a una práctica pedagógica aún tradicional, lineal y cerrada, poco (re) constructiva; no pudieron identificar dificultades de aprendizaje de sus alumnos y (re)planificar las acciones de enseñanza; e implementaron propuestas exentas de una perspectiva histórico-social y que vinculara Ciencia/Técnica/Sociedad/Ambiente, lo cual significó que las profesoras no comprendieron a la alimentación como un conocimiento de naturaleza histórica y social.

El trabajo de Izquierdo Aymerich et al. (2013) aborda la educación alimentaria para caracterizar modelos sobre alimentación de docentes y contrastarlos con los de especialistas de diversas disciplinas. En el marco de una investigación cualitativa identifican, a través de mapas 
conceptuales y la planificación de secuencias didácticas, los modelos puestos en juego por docentes para enseñar esta temática. Los modelos de los especialistas, reconstruidos a través de encuestas y entrevistas, evidencian que la alimentación es un fenómeno complejo y multidimensional, que incluye lo sociocultural. Los expertos señalan una tendencia a medicalizar la alimentación, dar un mayor enfoque biológico y sugieren rescatar los conocimientos históricos. En el análisis de los modelos docentes hallan resultados limitados y parciales en información y un mayoritario enfoque nutricional-sanitarista. A partir del estudio, plantean diseñar estrategias de acompañamiento a docentes, para la construcción "de islotes interdisciplinarios de racionalidad y razonabilidad”. (IZQUIERDO AYMERICH et al., 2013, p. 256).

Desde el campo de la formación inicial de docentes en Educación para el Consumo Sostenible, Sánchez, Conde y Garrido (2014) analizan propuestas didácticas elaboradas por futuros docentes de Educación Infantil, antes de abordar el tema en la asignatura Educación para la Salud. Su estudio se centra en cuatro ámbitos que consideran propios de la educación para el consumo desde la alimentación: aspectos relacionados con la salud, sociales, medioambientales y económicos. Evidencian que este alumnado realiza propuestas didácticas que se centran en aspectos relacionados con la salud, como la clasificación de alimentos en saludables o perjudiciales y la pirámide alimentaria, y tratan muy poco la incidencia del consumo alimentario sobre aspectos sociales, medioambientales o económicos.

Las propuestas analizadas evidencian, por un lado, una progresiva complejización de la mirada sobre la temática alimentación que, partiendo desde un enfoque más ligado a la Biología, van enriqueciendo y formulando recomendaciones para su tratamiento en ámbitos educativos con perspectivas multirreferenciadas e interdisciplinares. Por otro lado, señalan la necesidad de profundizar la formación docente referida a la temática.

\section{Descripción y fundamentación de la propuesta didáctica}

Si bien la temática alimentación no se encuentra explícitamente prescripta en el Diseño Curricular del Nivel Inicial ${ }^{6}$ de la Provincia de Buenos Aires (Argentina), suele ser abordada por las docentes, quienes la consideran entre los contenidos del bloque "El cuidado de la salud y el ambiente". En nuestra experiencia en espacios de formación docente en ejercicio para este nivel, al indagar acerca de las prácticas de enseñanza de contenidos vinculados a la salud, hemos identificado que las profesoras suelen trabajarlos a través de proyectos centrados en la enseñanza de la nutrición, de hábitos de conducta como la higiene personal, bucal y de manos; y en la prevención de enfermedades y accidentes, coincidentemente con Sánchez, Conde y Garrido (2014). Construidas en torno a conocimientos técnicos, estas propuestas generalmente incluyen la intervención de especialistas y el uso de materiales de campañas de difusión centralizadas. En ellas, la salud suele conceptualizarse como un fenómeno individual, identificable por la ausencia de enfermedad y regulado por un cuerpo de expertos dentro del sistema biomédico. Las propuestas que se implementan en el aula sobre alimentación están inspiradas en

${ }^{6}$ Educación Infantil, 0-5 años. 
programas nutricionales de organismos internacionales que, desde una perspectiva biomédica de la salud, proveen información y consejos sobre la cantidad y la composición de la comida "sana", la regulación del peso y la prevención de enfermedades. Las docentes cuentan, además, con una profusión de ofertas editoriales y materiales de divulgación que brindan información respecto de la necesidad fisiológica de nutrientes, basados en un modelo unificado y universal de cuerpo humano. De esta manera, quedan de lado los saberes y prácticas de niños, niñas y sus familias, así como la mirada compleja y contextualizada que el Diseño Curricular propone para el abordaje de la Educación en Salud.

En base a este diagnóstico, elaboramos e implementamos un curso para docentes en ejercicio que se planteó como objetivos: reflexionar sobre la enseñanza de la promoción de la salud construyendo posicionamientos críticos; caracterizar participativamente la enseñanza de temáticas vinculadas a la Salud en el nivel de Educación Inicial; problematizar la noción escolarizada de alimentación; construir una lectura situada y empíricamente informada sobre los contextos de actuación de las cursantes, específicamente con relación a sus prácticas alimentarias; construir marcos conceptuales en torno a la alimentación que permitan revisar la propia práctica docente; vivenciar la construcción colaborativa de conocimiento a partir de metodologías participativas; y diseñar proyectos educativos situados, comprometidos y en relación a los intereses y problemáticas de las comunidades escolares, orientados a promover la participación. Desde nuestra perspectiva, estos objetivos aportarían herramientas para la construcción de un posicionamiento crítico sobre la temática, comprometido con la realidad contextual concreta y compleja en la que las profesoras se desempeñaban.

Dicho posicionamiento crítico debía estar basado en un enfoque multidimensional asumido en el curso para el tratamiento de la alimentación, que considera no sólo la dimensión biomédica, sino también la socio-cultural y la político-económica. Así, entendemos a la alimentación como una práctica social compleja, comprendida y vivenciada de modos diversos por las personas y comunidades en el intento de lograr satisfacer sus necesidades vitales y como parte de su modo de vida y sentido de bienestar. Desde esta perspectiva, interrogantes tales como qué comemos, dónde, cuánto, cómo, cuándo y con quién se responden, no sólo en función de regular los cuidados del cuerpo, sino dentro de un entramado simbólico que expresa y recrea sentidos y prácticas elaborados en el seno de una cultura, y que por tanto construyen identidad. Como señalan estudios antropológicos

Así como no comemos calorías sino comida, tampoco comemos productos sino platos [...]. Es en la transformación doméstica de los productos de la canasta en platos de comida donde se juega la estrategia de consumo. Ya que es el lugar donde lo comestible se transforma en comida porque implica prácticas concretas, uso de tecnologías específicas y representaciones acerca de la vida, la comida y el cuerpo que indican dónde, cómo y quién debe comer qué, marcando diferencias sociales, sexuales y etarias. (AGUIRRE, 2004, p. 7).

En las sociedades actuales predomina una visión medicalizada de la dieta (GRACIA -ARNAIZ, 2007). Esto supone: universalizar un modelo de cuerpo, medible de acuerdo a parámetros físicos, como el índice de masa corporal, generalizados a partir de registros de pobla- 
ciones particulares; reducir a las personas a cuerpos-máquina que hay que nutrir adecuadamente; desconocer la diversidad sociocultural (o valorarla negativamente) tendiendo a homogeneizar; y centrar la responsabilidad de la alimentación en lo individual, dejando de lado condicionantes sociales, políticos y económicos. Dichos condicionantes se pondrían en evidencia a partir del tratamiento explícito de una dimensión político-económica, que tenga en cuenta relaciones de poder, etnia, clase y género.

En cuanto a los contenidos considerados en el curso, la dimensión biomédica incluyó aspectos biológicos de la alimentación: tipos de alimentos, nutrientes, dietas y sus representaciones gráficas (pirámides, óvalos, tablas); medicalización de la dieta y sus consecuencias. La dimensión socio-cultural abarcó la consideración de costumbres (por ejemplo: consumo y tipos de alimentos por sector social y comensalidad), hábitos y tradiciones. La dimensión económico-política se enfocó en el acceso a los alimentos, el derecho a la alimentación, y los conceptos de seguridad y soberanía alimentaria. Si bien para el análisis de situaciones presentamos estas tres dimensiones de manera diferenciada, en la práctica aparecieron entrelazadas.

El curso se implementó durante 2013, abarcó siete encuentros quincenales de tres horas cada uno, con actividades no presenciales entre ellos. Las docentes participantes fueron 46 profesoras de Educación Infantil en actividad, con distintos grados de experiencia, que concurrían voluntariamente a este espacio. Se desempeñaban en instituciones de gestión pública (y algunas también en otras de gestión privada) en la ciudad de La Plata y localidades aledañas, a las que concurren niños y niñas de condiciones socio-económicas y procedencias culturales diversas. El equipo docente a cargo incluyó a las autoras de este artículo, con diferentes formaciones de base (Didáctica de las Ciencias Naturales, Biología, Educación Ambiental, Teatro, Historia del Arte) y con la posibilidad de aportar distintas estrategias de trabajo y perspectivas disciplinares de análisis, a partir de un desafío de articulación interdisciplinaria.

Los núcleos temáticos trabajados fueron:

1. Concepciones de salud: consideramos la salud como derecho humano y como construcción social, compleja e histórica, y por lo tanto dinámica, atravesada por diferentes dimensiones (socio-cultural, política-económica, biológica, médica, psicológica, ambiental). Asumimos un enfoque de promoción de la salud, lo cual implica superar la visión de la población como mera destinataria pasiva y receptora de programas educativos desarrollados por profesionales. La promoción de la salud

[...] solicita el fortalecimiento de la acción comunitaria en la fijación de prioridades, toma de decisiones, elaboración y puesta en marcha de estrategias de planificación para alcanzar un mejor nivel de salud [...] trasciende la idea de formas de vida sana para incluir las condiciones y requisitos para la salud: la paz, la vivienda, la educación, la alimentación, la renta, un ecosistema estable, los recursos sostenibles, la justicia social y la equidad. (GÁLVEZ; IRAZOLA, 2006, p. 15).

2. alimentación: reconociendo a la alimentación como un aspecto de la salud en concordancia con el enfoque propuesto, estructuramos el desarrollo de la temática en torno a las dimensiones biomédica, sociocultural y político-económica. 
3. Enseñanza por proyectos: enfoque de enseñanza propuesto por el Diseño Curricular del Nivel Inicial, apropiado para una perspectiva multidimensional de la alimentación.

En el Cuadro 1 sintetizamos la secuencia de actividades y temáticas desarrolladas.

La modalidad de trabajo del curso incluyó: la recuperación y valoración de los saberes de las participantes; el trabajo en grupos en función de aprender colaborativamente, revisar los saberes, poner en juego la diversidad de miradas en el trabajo conjunto; la utilización de estrategias que recuperaran lo vivencial (como dramatizaciones); la presentación a través de distintos dispositivos de perspectivas teóricas sobre las temáticas abordadas (exposiciones orales y lecturas); la evaluación compartida sobre el proceso del curso en función de realizar ajustes;

Cuadro 1. Temáticas y secuencia de actividades desarrolladas

\begin{tabular}{|l|l|}
\hline \multicolumn{1}{|c|}{ Clase y tema } & \multicolumn{1}{c|}{ Secuencia de actividades } \\
\hline Clase 1: Salud & $\begin{array}{l}\text { - Diagnóstico colectivo sobre la enseñanza de salud en el Nivel Inicial. } \\
\text { - Explicitación de concepciones de salud propias. } \\
\text { - Exposición teórica de definiciones sobre salud y conceptualización. } \\
\text { - Tarea extra-clase: reflexión individual y presentación escrita sobre los aportes a } \\
\text { su práctica de la nueva concepción de salud construida colectivamente. }\end{array}$ \\
\hline $\begin{array}{l}\text { Clase 2: Primera } \\
\text { aproximación a } \\
\text { la Alimentación }\end{array}$ & $\begin{array}{l}\text { - Torbellino de ideas para la explicitación de nociones internalizadas sobre } \\
\text { alimentación. } \\
\text { - Identificación de dimensiones que atraviesan el tema (biomédica, socio-cultural y } \\
\text { económico-política). } \\
\text { - Trabajo grupal de análisis de las dimensiones, a partir de imágenes de familias de } \\
\text { distintos países y sus consumos alimentarios semanales7. } \\
\text { - Tarea extra-clase: recuperación de planificaciones de clases propias previas sobre } \\
\text { alimentación. }\end{array}$ \\
\hline $\begin{array}{l}\text { Clase 3: } \\
\text { Dimensión } \\
\text { biomédica de la } \\
\text { Alimentación }\end{array}$ & $\begin{array}{l}\text { - Presentación en plenario de análisis grupales desde el punto de vista biomédico } \\
\text { de las fotografías de familias de distintos países. } \\
\text { - Clasificación de tipos de alimentos y nutrientes. } \\
\text { - Análisis grupal de la dieta diaria de una integrante, a partir de diversas } \\
\text { clasificaciones y representaciones. } \\
\text { - Conceptualización acerca de qué implica una dieta saludable e inicio de la } \\
\text { discusión sobre medicalización de la dieta. } \\
\text { - Tarea extra-clase: lecturas sobre medicalización de la dieta (GRACIA-ARNAIZ, } \\
\text { 2007) y la dimensión socio-cultural de la alimentación (AGUIRRE, 2004). }\end{array}$ \\
\hline
\end{tabular}

\footnotetext{
${ }^{7}$ Información y fotografías disponibles en: < http://marcianosmx.com/hungry-planet-las-comidas-del-mundo/>. Acceso el: 15 oct. 2015.
} 
Cuadro 1. continuación

\begin{tabular}{|c|c|}
\hline Clase y tema & Secuencia de actividades \\
\hline $\begin{array}{l}\text { Clase 4: } \\
\text { Dimensión } \\
\text { socio-cultural de } \\
\text { la Alimentación }\end{array}$ & $\begin{array}{l}\text { - Puesta en común de las lecturas y exposición teórica sobre medicalización de la } \\
\text { dieta y consumo y tipos de alimentos por sector social y comensalidad. } \\
\text { - Elaboración, puesta en escena y análisis de dramatizaciones sobre experiencias } \\
\text { reales acerca de usos, costumbres y diferencias culturales en la Educación Infantil. } \\
\text { - Exposición teórica acerca de concepciones de la diversidad cultural } \\
\text { (DUSCHATZKY; SKLIAR, 2000). } \\
\text { - Tarea extra-clase: relevamiento sobre qué y cómo comen habitualmente sus } \\
\text { alumnas/os. Lectura extra-clase de un texto acerca de la dimensión económico- } \\
\text { política de la alimentación (APPLE, 2003). }\end{array}$ \\
\hline $\begin{array}{l}\text { Clase } 5: \\
\text { Dimensiones } \\
\text { socio-cultural } \\
\text { y económico- } \\
\text { política de la } \\
\text { Alimentación }\end{array}$ & $\begin{array}{l}\text { - Puesta en común y análisis de la lectura extra-clase. } \\
\text { - Reconstrucción grupal del circuito de producción, distribución, obtención y } \\
\text { consumo de diferentes alimentos, en base a la elección de tres ingredientes de un } \\
\text { plato asignado. } \\
\text { - Puesta en común de los análisis y reflexión acerca de los diversos circuitos y de } \\
\text { los conceptos de seguridad y soberanía alimentarias. } \\
\text { - Tarea extra-clase: comienzo de la elaboración grupal de un proyecto áulico sobre } \\
\text { alimentación. }\end{array}$ \\
\hline $\begin{array}{l}\text { Clase 6: } \\
\text { Enseñanza por } \\
\text { proyectos }\end{array}$ & $\begin{array}{l}\text { - Indagación de ideas previas acerca de la enseñanza por proyectos. } \\
\text { - Reconstrucción de criterios respecto de la elaboración de proyectos, a partir de } \\
\text { las indicaciones del Diseño Curricular y de LaCueva (1998). } \\
\text { - Trabajo grupal de elaboración de proyectos áulicos sobre Alimentación y entrega } \\
\text { de primera versión. } \\
\text { - Tarea extra-clase: redacción grupal de proyectos áulicos y elaboración de la } \\
\text { presentación oral. }\end{array}$ \\
\hline $\begin{array}{l}\text { Clase } 7 \text { : } \\
\text { Proyectos sobre } \\
\text { Alimentación }\end{array}$ & $\begin{array}{l}\text { - Socialización de producciones como instancia de aprendizaje/Evaluación de } \\
\text { aprendizajes logrados. } \\
\text { - Evaluación individual y colectiva de la propuesta de curso vivenciada. }\end{array}$ \\
\hline
\end{tabular}

Fuente: elaborado por las autoras.

la evaluación sobre la articulación entre lo trabajado en el curso y la práctica educativa real; y la reflexión acerca de la politicidad de la educación y del rol docente.

La implementación de esta modalidad se tradujo en el desarrollo de ciclos de trabajo que partieron de situaciones concretas de la práctica docente, la reflexión sobre ellas desde distintos marcos teóricos y la elaboración de alternativas para volver a la práctica. Una forma de regresar a la práctica fue la construcción colaborativa de proyectos áulicos en grupos de docentes, con el acompañamiento del equipo de formadoras. El proceso colaborativo de reflexión sobre las 
prácticas habituales para su mejoramiento se concretó a través de las tareas extra-clase solicitadas (Cuadro 1), y su puesta en común y discusión en el aula en distintos momentos del curso.

Para la evaluación de la propuesta y de los aprendizajes, implementamos diversas instancias e instrumentos individuales y grupales. Para la evaluación de la propuesta, la información fue relevada en una instancia presencial en la que se valoraron diversos aspectos, mientras que otros fueron valorados por las cursantes a través de un cuestionario respondido por correo electrónico. Para la evaluación sumativa del curso se solicitó la elaboración grupal de proyectos áulicos, fundamentados desde su conocimiento del contexto, la bibliografía trabajada y el Diseño Curricular, con actividades y productos esperados detallados.

\section{Encuadre metodológico}

Este trabajo pretende aportar al campo de la formación docente en CNAS, ya que constituye un análisis de una experiencia de formación (que incluyó la reflexión sobre la práctica entre docentes) acerca de una temática compleja y multidimensional, implementada y evaluada por sus participantes. La misma fue desarrollada en el contexto del Proyecto "Estudio de Representaciones y Prácticas en la Educación en Ciencias Naturales, Ambiente y Salud" (PERsPECNAS), cuyo principal objetivo es analizar prácticas educativas innovadoras y procesos de transformación de representaciones sociales respecto de tópicos específicos del área. Según Messina Raimondi (2008), una posibilidad, desde el punto de vista metodológico, de investigación sobre la formación docente es la sistematización de experiencias, “[...] la sistematización como un camino para crear saber pedagógico desde la experiencia, la deconstrucción de la experiencia, la producción de un saber colectivo, la crítica radical; la deconstrucción como un proceso de desmontar la realidad" (MESSINA RAIMONDI, 2008, p. 11).

La sistematización de experiencias educativas puede ser considerada como investigación cualitativo-crítica, en la cual se desarrollan simultáneamente los procesos de reconstrucción, interpretación y transformación de la práctica. En este sentido, la sistematización se vincula estrechamente con la investigación-acción en el aula, particularmente con aquélla que focaliza en el análisis de la propia práctica y la construcción de conocimiento para su mejora (KEMMIS; McTAGGART, 2005).

Para la sistematización de esta propuesta formativa, la base empírica se sustenta en: registros (etnográficos y audiograbaciones) de las clases desarrolladas ${ }^{8}$, planificaciones de las mismas, materiales utilizados y producciones de las docentes participantes a lo largo del proceso. La reconstrucción de la experiencia comenzó por la transcripción, organización y análisis cualitativo de registros audiograbados de clases y de producciones en clase y extra-clase individuales y grupales; la elaboración de bases de datos de respuestas a encuestas y cuestionarios y su tratamiento analítico cualitativo, a partir de su recorte por pregunta y por tema emergente.

\footnotetext{
${ }^{8}$ Agradecemos la realización de los registros a las cursantes de Didáctica de las Ciencias Naturales (Facultad de Humanidades y Ciencias de la Educación, Universidad Nacional de La Plata) participantes de esta experiencia.
} 
El análisis siguió las fases o momentos que plantea Bardin (1977): un pre-análisis que implicó la realización de lecturas superficiales, a partir de las cuales se comenzaron a identificar categorías generales; en una segunda fase, de "aprovechamiento del material", se realizaron operaciones de codificación, descomposición y enumeración, a partir del pre-análisis. La tercera fase fue de "tratamiento de resultados, inferencia e interpretación", en la cual se elaboraron ejes analíticos, que presentamos en la próxima sección.

\section{Análisis y discusión de resultados}

\section{Diagnóstico y caracterización de las prácticas habituales}

El curso comenzó con un diagnóstico realizado colectivamente, que nos permitió relevar el tratamiento de contenidos y el desarrollo de actividades relativos a la alimentación en la Educación Infantil, - aún cuando, como hemos dicho, no es contenido prescripto por el Diseño Curricular. Tanto al enumerar contenidos como al definir propósitos, las profesoras orientaban su práctica en un sentido preventivo y de enseñanza de hábitos (Cuadro 2), lo cual caracterizaría al nivel educativo. Se destacó el rol de profesionales, invitados a las aulas, como portadores del conocimiento validado y legítimo para el abordaje de las cuestiones de salud. Resultó también muy interesante la variedad de dispositivos descriptos por las profesoras al caracterizar sus modos habituales de tratamiento de estas temáticas.

Cuadro 2. Caracterización colectiva de prácticas habituales en la enseñanza de Salud en la Educación Infantil

\begin{tabular}{|c|c|c|c|c|}
\hline $\begin{array}{c}\text { Qué } \\
\text { se enseña }\end{array}$ & $\begin{array}{l}\text { Para qué } \\
\text { se enseña }\end{array}$ & $\begin{array}{l}\text { Quiénes } \\
\text { enseñan }\end{array}$ & $\begin{array}{l}\text { Quiénes } \\
\text { aprenden }\end{array}$ & $\begin{array}{c}\text { Cómo } \\
\text { se enseña }\end{array}$ \\
\hline $\begin{array}{l}\text { Alimentación } \\
\text { Contaminación } \\
\text { Cuidado del } \\
\text { cuerpo } \\
\text { Prevención de } \\
\text { accidentes } \\
\text { Prevención de } \\
\text { enfermedades } \\
\text { (vacunación) } \\
\text { Higiene del cuerpo } \\
\text { Higiene bucal } \\
\text { Hábitos de higiene } \\
\text { Actividad física }\end{array}$ & $\begin{array}{l}\text { Prevención } \\
\text { Informar } \\
\text { Cuidado del } \\
\text { otro } \\
\text { Cuidado de } \\
\text { uno mismo } \\
\\
\text { Estadística } \\
\text { Educar } \\
\text { Transmitir } \\
\text { experiencias }\end{array}$ & $\begin{array}{l}\text { Docente } \\
\text { Ida y vuelta } \\
\text { (Docentes y } \\
\text { Alumnos) }\end{array}$ & Docentes & $\begin{array}{l}\text { Comparaciones de alimentos } \\
\text { Talleres con familias } \\
\text { Carpetas viajeras } \\
\text { Conocer hábitos } \\
\text { Pirámide alimentaria } \\
\text { Experiencia directa } \\
\text { Talleres de cocina } \\
\text { Hábitos/merienda } \\
\text { Visita de profesional } \\
\text { Repetición de acciones/hábitos } \\
\text { Títeres, videos, cuentos, maquetas, } \\
\text { modelos } \\
\text { Comparaciones, imágenes } \\
\text { Normas de convivencia } \\
\text { Visita a la sala de primeros auxilios } \\
\text { Calendario de vacunación }\end{array}$ \\
\hline
\end{tabular}

Fuente: elaborado por las autoras. 
La reflexión sobre la enseñanza de la promoción de la salud construyendo posicionamientos críticos comenzó en el primer encuentro del curso. En dicha ocasión, a través del análisis de definiciones, elaboradas grupalmente sobre qué es la Salud en la Educación Infantil, y de producciones gráficas bajo la consigna: "Estar sano es...”, encontramos una lectura de la salud centrada en el individuo y atravesada, principalmente, por aspectos biomédicos y psicológicos. Las dimensiones que, desde nuestro punto de vista, estuvieron prácticamente ausentes fueron la político-económica y la socio-cultural. Éstas formarían parte de lo "no pensable" para las profesoras, en el marco de un habitus que oculta las relaciones de poder presentes en toda sociedad - coherente con una mirada hegemónica normalizadora (MENGASCINI et al., 2014).

\section{La problematización de la noción de alimentación}

Con relación al objetivo de problematizar la noción escolarizada de alimentación, las planificaciones habituales sobre el tema-solicitadas al inicio del curso- evidenciaron el énfasis puesto en la repetición de acciones/hábitos - por ejemplo durante la merienda -, las comparaciones de alimentos saludables y no saludables, la implementación de recetas en talleres de cocina, y el uso de la pirámide alimentaria. Para analizar la problematización lograda recuperamos el relevamiento de las nociones internalizadas sobre alimentación y su discusión, a través de los registros de las actividades y exposiciones a lo largo del curso.

Dichas nociones se explicitaron a través de un "torbellino de ideas" en torno a la palabra "alimentación”. Del torbellino surgieron 59 términos que clasificamos dentro de las tres dimensiones consideradas (aunque con notable predominio de la dimensión biomédica, más rica tanto en cantidad de términos como en aspectos considerados). Así, en relación con la dimensión sociocultural se mencionaron: "costumbres, cultura, folclore, cocinar es distinto de hacer de comer". Dentro de los aspectos político-económicos: "canasta básica, economía, educación, derecho". Respecto de la dimensión biomédica: "carbohidratos, dieta, nutrientes" (que hacen referencia a aspecto biológicos y nutricionales); "crecimiento, cuidado, acto de amor, contención, adicción, angustia oral" (que refieren a aspectos relacionados a lo psicoafectivo); "desnutrición, enfermedades, higiene y obesidad" (que refieren a aspectos médicos normativos).

Como se menciona en el Cuadro 1, en la misma clase se realizó el análisis de las comidas de familias de distintos países representadas en fotografías. Según las discusiones registradas, uno de los grupos fácilmente acordó qué es lo variado, nutritivo y saludable de una dieta, pero revisó estas ideas atendiendo a diversidad de costumbres (tomando como ejemplo una dieta de una familia de un país oriental) y a elecciones personales (dieta vegetariana). Respecto de la dimensión socio-cultural, además de las comidas presentadas en la fotografía, se tuvo en cuenta para la caracterización a la vivienda, su carácter urbano o rural, la vestimenta, la composición familiar, y se reflexionó acerca de las diferencias entre prácticas resultantes de costumbres o de necesidades (por imposibilidad de acceso a ciertos alimentos en distintas clases sociales y/o países). Respecto de la dimensión político-económica, se registraron diferencias vinculadas a limitaciones económicas y formas de acceso a los alimentos (por compra o producción propia). Otro grupo enmarcó el consumo de una de las familias representadas en las fotografías en el sistema capitalista.

La socialización de las producciones y su revisión colectiva permitió la explicitación de “ideales de nutrición”; el análisis de las mayores o menores posibilidades de consumo en los diversos 
países y familias y de la posibilidad de producción de los propios alimentos o el consumo de alimentos elaborados; y el reconocimiento de aspectos culturales, económicos y políticos en las prácticas alimentarias. Ante la pregunta acerca del sentido otorgado a los términos "equilibrada", "nutritiva", "saludable", "variada", respecto de la alimentación de las diversas familias, las cursantes especificaron: "equilibrado es un poco de cada cosa", "que no sea mucha proteina o mucho carbohidrato"; saludable es "que aporta nutrientes, que es algo balanceado que tiene un equilibrio", "que es rica en vitaminas, proteinas", "que tengan todos los nutrientes necesarios para el organismo". En ese contexto, los usos asignados por las profesoras a la pirámide nutricional fueron como "indicador", "para tener una noción", "sería como una plataforma de alimentos, pero uno tiene que hacer una dieta equilibrada, conforme a las necesidades que tiene", "como un paradigma, un modelo". Afirmaron que "la pirámide es equilibrada, pero no con todos funciona". La introducción de otras formas de representación como el óvalo nutricional (la "gráfica de la alimentación saludable") (LEMA; LONGO; LOPRESTI, 2003) y una tabla relacionando tipos de alimentos (energéticos, reguladores y plásticos o estructurales), grupos (generalmente considerados en las pirámides nutricionales), nutrientes y ejemplos, que ofrecimos las formadoras, permitió identificar las diferencias entre ellas. Las profesoras valoraron que la tabla "se aboca más a la calidad y al aporte especifico que nos da cada alimento".

En cuanto a los aspectos político-económicos de las prácticas alimentarias, no se profundizó en análisis que abordaran las relaciones de poder entre países, o una lectura política que explicara las relaciones de dominación (aún cuando fotografías ofrecidas permitían visualizar grandes desigualdades en el acceso a alimentos).

Para abordar la dimensión socio-cultural de la alimentación, durante la cuarta clase (Cuadro 1), se propuso la escenificación grupal de situaciones escolares vividas, que permitieran mostrar las representaciones sobre "lo cultural" portadas por las maestras. Los grupos dramatizaron: las diferencias de actitudes de familias en instituciones de gestión pública (mayor participación y compromiso) y de gestión privada (mayor interés en su "inversión" en la educación de sus niños/as); la falta de sostenimiento de hábitos "saludables", como el desayuno, y sus efectos en niños/as (responsabilizando a las familias); las prácticas tradicionales de sanación de malestares gástricos (como el "empacho"), reconocidos por las familias pero no por el sistema educativo, que requiere certificación de alta médica para reincorporar a los niños/as; y una situación en la que una niña inmigrante llamaba "tía" a la profesora, provocando risas y burlas entre sus compañeros/as. En este último caso, en el que se evidenció más claramente qué es lo que se estaba entendiendo por diversidad cultural, la intervención de la profesora en la escena apuntó a explicar a los niños y niñas que no debían burlarse, porque la niña "venía de otro lado" y recomendó "tengámosle paciencia, hasta que se acostumbre a nuestras costumbres". El grupo explicó: "nosotras justamente quisimos mostrar que los que vienen de afuera tienen que integrarse a la diversidad cultural, ellos se tienen que integrar a nosotros”. Pusimos en tensión tales afirmaciones a la luz de las conceptualizaciones acerca de la diversidad cultural planteadas por Duschatsky y Skliar (2000).

Luego de la lectura del texto de Apple (2003) sobre la relación entre etnia, clase y formas de producción de alimentos, el análisis de la dimensión económico-política, realizada en grupos a través de la reconstrucción de la "ruta" de componentes de diversos platos (quinta clase, Cuadro 1), permitió visualizar los grados y formas de problematización de esta perspectiva. Dos de los grupos produjeron esquemas que consideraron los aspectos sociales y legales vinculados a las formas de producción, circulación y acceso a los alimentos; otros dos grupos mostraron distintas formas de producción; otros dos señalaron en detalle las etapas en dicho circuito; y el 
último de los grupos produjo el esquema más simplificado, sin evidenciar profundización en ninguno de los aspectos analizados. En la conceptualización final sobre esta dimensión analítica, las formadoras ahondamos en los diversos circuitos y sus consecuencias socio-ambientales (particularmente respecto de la producción de organismos genéticamente modificados).

\section{Elaboración de proyectos educativos situados a partir de la revisión de la práctica}

Un objetivo central y vertebrador del curso fue revisar la propia práctica docente. Para su consecución desarrollamos toda la secuencia de actividades de clase y extra-clase (Cuadro 1). Si bien a través de ellas pudimos seguir los diversos procesos de construcción y revisión, las producciones parciales y finales de las cursantes, así como sus autoevaluaciones al finalizar la experiencia constituyeron las fuentes más informativas respecto de lo logrado.

Para propiciar la producción de proyectos áulicos basados en el conocimiento de las prácticas alimentarias de los sujetos con los que trabajaban, se solicitó como tarea extra-clase, promediando el curso y luego de abordar la dimensión socio-cultural de la alimentación, la realización de un relevamiento, entre los niños, niñas y familias, respecto de qué y cómo comían habitualmente (con quiénes, en qué momentos, quién/es cocinaba/n, entre otros aspectos). Esta actividad también requería operativizar herramientas conceptuales ya presentadas. Al tratarse de niños y niñas de muy corta edad (inclusive lactantes), la tarea de relevamiento ofreció dificultades para algunas profesoras, que debieron recurrir a la realización de encuestas escritas a las familias. Otras, trabajadoras en instituciones de jornada completa, en las que se brindaban varias comidas diarias, optaron por describir y analizar las prácticas alimentarias institucionales. En otros casos, el intercambio con niñas y niños permitió a las profesoras visualizar informaciones novedosas, como las diferencias entre los almuerzos ofrecidos por madres y padres ("comidas rápidas") y por abuelos y abuelas (más elaboradas y caseras). Finalmente, en otros casos, confirmaron sus observaciones previas acerca de prácticas familiares en lo referido a la alimentación ("no mantienen un equilibrio de nutrientes y un aporte óptimo de vitaminas y minerales, sino por el contrario, abundan las grasas saturadas e hidrogenadas"). Estas informaciones ofrecieron un panorama de la diversidad de prácticas y contribuyeron en distintos grados a la generación de los proyectos áulicos que las docentes presentaron como producción final.

Los trabajos finales grupales (por entre dos y cinco profesoras) fueron diez.

En la mayoría de los proyectos la dimensión socio-cultural se asoció a dispositivos y contenidos que formaban parte de las prácticas docentes habituales (como la indagación de prácticas familiares de alimentación, la preparación de alimentos y la elaboración de "recetarios viajeros", con recetas aportadas por las diferentes familias). Dos de los trabajos rescataron a la "comensalidad" como un concepto abordable con los niños en el aula. Esta sería una puerta de entrada elegida por las docentes para incorporar la dimensión en este nivel educativo. Otra puerta de entrada, que surge del análisis de los trabajos finales, sería una perspectiva histórica, a través de "indagar con los alumnos algún aspecto de la vida cotidiana de poblaciones originarias de nuestro territorio en el pasado y el presente", comparando comidas y alimentos de estos pueblos con lo que hoy consumimos y cómo lo obtenemos. En siete trabajos se trató algún aspecto de la dimensión económico-política, centralmente la reflexión sobre oficios y trabajos, los circuitos de distribución y consumo de alimentos, y visitas a lugares de producción y venta. Destacamos 
Cuadro 3. Identificación de actividades presentes en los proyectos docentes, por dimensión de la Alimentación

\begin{tabular}{|c|c|c|c|c|c|c|c|c|c|c|c|c|c|c|}
\hline \multirow[t]{2}{*}{ Título del trabajo } & \multicolumn{3}{|c|}{$\begin{array}{l}\text { Dimensión } \\
\text { Socio- } \\
\text { cultural }\end{array}$} & \multicolumn{5}{|c|}{$\begin{array}{c}\text { Dimensión } \\
\text { Económico-política }\end{array}$} & \multicolumn{6}{|c|}{ Dimensión Biomédica } \\
\hline & IPF & RV & PA & PP & VCF & VP & ROT & RCPC & NH & EHA & CA & $\mathbf{E}$ & COA & CMS \\
\hline La panadería del barrio & & & $\mathrm{x}$ & & $\mathrm{x}$ & $\mathrm{x}$ & $\mathrm{x}$ & & & & & & $\mathrm{x}$ & \\
\hline $\begin{array}{l}\text { La nutrición y los hábitos } \\
\text { de higiene para crecer } \\
\text { sanos y fuertes }\end{array}$ & & $\mathrm{x}$ & & & $\mathrm{x}$ & & $\mathrm{x}$ & & $\mathrm{x}$ & $\mathrm{x}$ & $\mathrm{x}$ & $\mathrm{x}$ & $\mathrm{x}$ & \\
\hline Dieta, consumo y hábitos & $\mathrm{x}$ & & $\mathrm{x}$ & & & & & & $\mathrm{x}$ & $\mathrm{x}$ & $\mathrm{x}$ & & $\mathrm{x}$ & \\
\hline A comer mejor & $\mathrm{x}$ & & & & $\mathrm{x}$ & & $\mathrm{x}$ & & & $\mathrm{x}$ & $\mathrm{x}$ & $\mathrm{x}$ & & $\mathrm{x}$ \\
\hline La Alimentación & $\mathrm{x}$ & & $\mathrm{x}$ & & & & & & $\mathrm{x}$ & $\mathrm{x}$ & $\mathrm{x}$ & & & \\
\hline ¿Cómo nos alimentamos? & $\mathrm{x}$ & $\mathrm{x}$ & $\mathrm{x}$ & & & & $\mathrm{x}$ & & & $\mathrm{x}$ & $\mathrm{x}$ & & & $\mathrm{x}$ \\
\hline Una alimentación variada & $\mathrm{x}$ & $\mathrm{x}$ & $\mathrm{x}$ & & & & & & & $\mathrm{x}$ & $\mathrm{x}$ & $\mathrm{x}$ & & $\mathrm{x}$ \\
\hline $\begin{array}{l}\text { ¿Qué puede comer el niño } \\
\text { celíaco? }\end{array}$ & $\mathrm{x}$ & & $\mathrm{x}$ & & & & & $\mathrm{x}$ & $\mathrm{x}^{\mathrm{a}}$ & & $\mathrm{x}$ & $\mathrm{x}$ & & \\
\hline $\begin{array}{l}\text { La huerta orgánica del } \\
\text { jardín }\end{array}$ & $\mathrm{x}$ & $\mathrm{x}$ & $\mathrm{x}$ & $\mathrm{x}$ & & $\mathrm{x}$ & $\mathrm{x}$ & $\mathrm{x}$ & & & $\mathrm{x}$ & & & \\
\hline $\begin{array}{l}\text { La alimentación: una } \\
\text { mirada a través de sus } \\
\text { dimensiones }\end{array}$ & $\mathrm{x}$ & $\mathrm{x}$ & $\mathrm{x}$ & $\mathrm{x}$ & & & $\mathrm{x}$ & $\mathrm{x}$ & $\mathrm{x}$ & & $\mathrm{x}$ & $\mathrm{x}$ & $x^{b}$ & \\
\hline
\end{tabular}

Siglas utilizadas: IPF: Indagación de prácticas familiares; RV: Recetario viajero; PA: Preparación de alimentos; PP: Producción propia; VCF: Visita a comercios y ferias; VP: Visitas a lugares de producción; ROT: Reflexión sobre oficios y trabajos en la alimentación; RCPC: Reflexión sobre circuitos de producción y consumo; NH: Enseñanza de normas de higiene; EHA: Enseñanza de hábitos alimentarios; CA: Clasificación de alimentos; E: Conferencia de especialista; COA: Conservación de alimentos; CMS: Construcción de menúes saludables.

${ }^{a}$ Normas de elaboración para celíacos; ${ }^{\mathrm{b}}$ Desde una perspectiva histórica

Fuente: elaborado por las autoras.

finalmente el enriquecimiento de la fundamentación de las propuestas en general. En términos generales, aún cuando no se logró el enmarcamiento en las relaciones de dominación y los sistemas económicos en sus fundamentaciones, las propuestas incluyeron la reflexión sobre la alimentación como derecho humano, el análisis de diversos circuitos de producción y acceso, 
llegando a plantear el autoanálisis de las instituciones educativas con relación a estas prácticas?. Estas producciones estarían evidenciando la apropiación, aunque de manera limitada, de una perspectiva crítica para el abordaje de la temática alimentación.

Respecto de la participación de familias y comunidades, ocho trabajos la incluyeron en diversos grados, que variaron desde la indagación de prácticas a través de encuestas y la participación de las familias en la elaboración de recetarios viajeros hasta la visibilización de problemáticas sociales y ambientales (trabajo infantil, uso de pesticidas) y la producción en huertas orgánicas comunitarias para consumo familiar e institucional de los productos. Dos proyectos resultaron descontextualizados, ya que partieron de una mirada más evaluativa que comprensiva de las prácticas familiares.

Luego del trabajo de reflexión y conceptualización compartida a lo largo del curso, las profesoras expresaron por escrito sus consideraciones acerca de los aportes de las revisiones desarrolladas a su práctica: "Considero importante este trabajo ya que al ser un tema abordable desde distintas dimensiones, que involucra a personas e instituciones, es necesaria la profundización de los conocimientos para [...] buscar el compromiso de todos, en un tema tan abarcativo, transversaly fundamental como la salud".

Respecto a las revisiones logradas, la mayoría de las docentes indicó que el curso brindó aportes conceptuales, específicamente referidos a la complejidad y multidimensionalidad de la alimentación: "El principal cambio es correrse de esa mirada simplista referida a la salud y alimentación que por lo general se tiene. Y abordar el tema de un modo global y multidimensional”. Numerosas profesoras consideraron que pudieron reflexionar y replantearse su práctica áulica diaria y sus planificaciones: "Cuando terminé el curso tomé una planificación mía vieja de alimentación y me di cuenta lo pobre que era".

\section{Vivencia de una construcción colaborativa de conocimiento}

Con la intención de que las profesoras vivenciaran procesos de construcción colaborativa de conocimiento se implementaron diferentes actividades grupales, tales como: dramatización de situaciones en las que aparecieran usos, costumbres y diferencias culturales en la Educación Infantil; construcción de producciones gráficas y definiciones grupales sobre salud; análisis conjunto de fotografías; entre otras. El trabajo en pequeños grupos ocupó alrededor del $50 \%$ del tiempo de cada encuentro y se desarrolló con la coordinación de las formadoras, que nos posicionamos manteniendo un vínculo de proximidad, facilitador del aprendizaje, pero con la distancia que permitiera que los grupos siguieran sus propios procesos. Para ello, las formadoras alternábamos las funciones de comunicar o aclarar consignas de las tareas; generar preguntas para orientar la discusión; reformular explicaciones no comprendidas luego de alguna exposición teórica; administrar los tiempos; y controlar la realización de actividades. Esa proximidad y evaluación permanente de comprensiones logradas por las profesoras permitía, además, un ajuste constante de las clases - realizado in situ a partir de breves discusiones y decisiones entre miembros del equipo - introduciendo exposiciones y discusiones generales, cuando las considerábamos necesarias, o variando la distribución de tiempos destinados a cada

\footnotetext{
${ }^{9}$ En el Proyecto anual institucional "La Alimentación: una mirada a través de sus dimensiones".
} 
tarea. Así, el propio funcionamiento colaborativo del equipo formador obraba como modelo de actuación pedagógica. Por otro lado, los análisis y discusiones acontecidos en los grupos siempre debían ser plasmados en productos que evidenciaran la diversidad de ideas o posicionamientos, en caso de que existieran. El trabajo grupal fue considerado por las cursantes “ $m u y$ interesante ya que pudimos escuchar y compartir otras realidades". También valoraron positivamente la realización grupal del trabajo final, destacando que había resultado "acorde e interesante de realizar en grupo, porque asi cada uno puede enriquecerse con las ideas y aportes de los otros".

\section{Consideraciones finales}

La sistematización de experiencias educativas pretende la reconstrucción, desde una mirada más densa, y la identificación de la lógica y nudos problemáticos de las mismas. En nuestro caso, la implementación de ciclos de trabajo en el proceso de formación docente en ejercicio, en los que el eje fue la práctica, convertida en praxis - a partir de la reflexión e incorporación de nuevos marcos teóricos - fue la lógica prevalente. En torno a esta tarea concreta de reflexión, el curso ofreció además el espacio para la constitución de una comunidad profesional compuesta por las profesoras y por nosotras como formadoras. Asimismo, el constante entramado de dispositivos que focalizaban en alguna de las dimensiones de la temática para luego ampliar el foco y develar los entrecruzamientos con las demás dimensiones, permitió evidenciar y poner en escena la multidimensionalidad y complejidad de la alimentación como objeto de estudio. Los ciclos de trabajo, el espacio de la comunidad y el entramado de dispositivos promovieron la elaboración de aprendizajes y productos que mostraron un relevante grado de avance respecto a las prácticas habituales en la Educación Infantil. Consideramos así que la propuesta constituyó un escenario "para romper el aislamiento del trabajo de cada profesor o profesora, crear las condiciones para enfrentarse a los retos siempre renovados de la educación y favorecer el interés por la docencia como tarea abierta y creativa" (VILCHES; GIL, 2010, p. 664).

La representación sobre Alimentación que apareció inicialmente al considerar sólo las prácticas habituales (la que hemos llamado noción escolarizada) evidenció una base fuerte en aspectos normativos. Las diversas actividades propuestas durante el curso para poner en cuestión dicha noción fueron útiles para producir una representación colectiva más rica, compleja y multidimensional. Tanto los resultados plasmados en las producciones finales de las profesoras cuanto sus autoevaluaciones, mostraron también un cambio relevante respecto a la situación inicial a diferencia de lo encontrado por Galvão y Praia (2009). En el caso que aquí analizamos, las profesoras hallaron diversas puertas de entrada para anclar en su práctica las dimensiones teóricas abordadas (a partir de la comensalidad, la perspectiva histórica y el abordaje de diferentes vías de distribución y consumo de alimentos).

Como nudo problemático, luego de este análisis, observamos la dificultad de las profesoras para profundizar en la dimensión político-económica. Los abordajes habituales de la temática Alimentación tienen un gran peso en la dimensión biomédica, como lo revela la literatura del campo y las prácticas docentes revisadas. Esta característica evidenciaría una concepción del conocimiento científico como universal y objetivo, sustento de normas para todas las poblaciones y culturas. Tal posicionamiento dificultaría la adopción de perspectivas pedagógicas críticas que, necesariamente, implican el reconocimiento de condicionamientos so- 
cio-políticos, económicos y culturales. Durante el desarrollo del curso, parcialmente y en algunos casos, esta dificultad logró superarse. Sin embargo, se requeriría el ajuste de algunas consignas (por ejemplo, en la actividad que proponía el análisis de fotografías) y una mayor orientación y profundización en el debate a fin de promover una apropiación más amplia y consolidada de esta mirada crítica. Otro aspecto a tener en cuenta sería el acompañamiento más sostenido en el tiempo de la elaboración de los proyectos áulicos, enfatizando la importancia de que los mismos consideren las tres dimensiones que atraviesan la temática.

Aun sosteniendo que para lograr transformaciones profundas y duraderas de la enseñanza se requiere la continuidad de un trabajo colectivo de reflexión crítica sobre la práctica, entendemos que propuestas como la presentada en este trabajo contribuyen a fortalecer el desarrollo profesional docente.

\section{Referencias}

AGUIRRE, P. Los alimentos rendidores y el cuerpo de los pobres. [UNLP, 2004].

Disponible en: <http://www.unlp.edu.ar/uploads/docs/Los $\% 20$ Alimentos $\% 20$

Rendidores $\% 20 \mathrm{y} \% 20 \mathrm{el} \% 20$ Cuerpo $\% 20 \mathrm{de} \% 20 \operatorname{los}_{0} \% 20$ Pobres $\% 20$ Patricia $\% 20$ Aguirre. $\% 20$ los $\% 20$ alimentos $\% 20$ rendidores $\% 20 y \% 20$ el\%20cuerpo.pdf>. Acceso el: 3 mar. 2013.

APPLE, M. W. Comiendo papas fritas baratas. Docencia, Santiago de Chile, n. 20, p. 47-55, 2003.

BARDIN, L. Análisis de contenido. 2. ed. Madrid: Akal, 1977.

DEL CAMPO, M. L.; VARA MESSLER, M.; NAVARRO, A. Educación alimentarianutricional en la enseñanza primaria municipal de Córdoba: una experiencia en investigaciónacción participativa. Diaeta, Buenos Aires, v. 28, n. 132, p. 15-22, 2010.

DUSCHATZKY, S.; SKLIAR, C. La diversidad bajo sospecha: reflexiones sobre los discursos acerca de la diversidad y sus implicancias educativas. Cuaderno de Pedagogía, Rosario, v. 4, n. 7, p. 1-13, 2000. Disponible en: < http://www.porlainclusionmercosur.educ. ar/documentos/Ladiversidadbajosospecha.pdf>. Acceso el: 17 nopv. 2015.

GALVÃO, V. S.; PRAIA, J. Construir com os professores do $2^{\circ}$ ciclo práticas letivas inovadoras: um projeto de pesquisa sobre o ensino do tema curricular 'Alimentação Humana'. Ciência \& Educação, Bauru, v. 15, n. 3, p. 631-645, 2009. Disponible en: <http://dx.doi.org/10.1590/S1516-73132009000300011>. Acceso el: 17 nopv. 2015.

GALVEZ, R.; IRAZOLA, J. El árbol de la transformación: manual de metodologías participativas para agentes de salud comunitaria. Buenos Aires: Medicusmundi, 2006.

GRACIA-ARNAIZ, M. Comer bien, comer mal: la medicalización del comportamiento alimentario. Salud Pública de México, México, v. 49, n. 3, p. 236-242, 2007. 
Cordero, S.; Mengascini, A.; Menegaz, A. et al.

IZQUIERDO AYMERICH, M. et al. Modelización del fenómeno de alimentación humana: de la mirada disciplinar a la multirreferencialidad. In: CONGRESO INTERNACIONAL SOBRE INVESTIGACIÓN EN DIDÁCTICA DE LAS CIENCIAS, 9., 2013, Girona. Actas... Girona: UAC, 2013. p. 252-258. Disponible en: <http://congres.manners.es/ congres_ciencia/gestio/creacioCD/cd/articulos/art_1009.pdf >. Acceso el: 29 oct. 2015.

KEMMIS, S.; McTAGGART, R. Participatory action research: communicative action and the public sphere. In: DENZIN, N. K.; LINCOLN, Y. S. (Ed.). The Sage handbook of qualitative research. 3rd. ed. Thousand Oaks: Sage, 2005. p. 559-603.

LACUEVA, A. La enseñanza por proyectos: ¿mito o reto? Revista Iberoamericana de Educación, Madrid, v. 16, p. 165-187, 1998.

LEMA, S.; LONGO, E.; LOPRESTI, A. Guías alimentarias para la población argentina. Buenos Aires: Asociación Argentina de Dietistas y Nutricionistas Dietistas: Ministerio de Salud, 2003.

MEMBIELA, P.; CID, M. Desarrollo de una unidad didáctica centrada en la alimentación humana, social y culturalmente contextualizada. Enseñanza de las Ciencias, Barcelona, v. 16, n. 3, p. 499-511, 1998.

MENGASCINI, A. et al. Más allá de la visión escolarizada de alimentación: resultados de una propuesta de formación en ejercicio con docentes de nivel inicial. En: ENCUENTROS DE DIDÁCTICA DE LAS CIENCIAS EXPERIMENTALES, 26., 2014, Huelva. Actas... Huelva: UH, 2014. p. 318-326.

MESSINA RAIMONDI, G. Formación “docente”: del control al saber pedagógico.

Docencia, Santiago de Chile, n. 34, p. 78-86, 2008. Disponible en: <http://www. revistadocencia.cl/pdf/20100731221204.pdf>. Acceso el: 29 oct. 2015.

RIVAROSA, A.; DE LONGHI, A. L. Aportes didácticos para nociones complejas en biología: la alimentación. Buenos Aires: Miño y Dávila, 2012.

ROSET ELÍAS, A.; VILADOT AGUAYO, R. La educación nutricional. Aula de Innovación Educativa, Barcelona, n. 137, p. 85-96, 2004.

SÁNCHEZ, J.; CONDE, M.; GARRIDO, A. Análisis de los contenidos de educación para el consumo alimentario propuestos por estudiantes del grado de educación infantil. In: XXVI ENCUENTROS DE DIDÁCTICA DE LAS CIENCIAS EXPERIMENTALES, 26., 2014, Huelva, 2014. Actas... Huelva: UH, 2014. p. 634-642.

VILCHES, A.; GIL, D. Máster de formación inicial del profesorado de enseñanza secundaria: algunos análisis y propuestas. Revista Eureka sobre Enseñanza y Divulgación de las Ciencias, Cádiz, v. 7, n. 3, p. 661-666, 2010.

Artigo recebido em 10/02/2015. Aceito em 22/09/2015.

Dirección para contacto: Calle 59, n. 789, (1900), La Plata, Buenos

Aires, Argentina. 